\title{
SIMPLICITY OF NEAR-RINGS OF TRANSFORMATIONS
}

\author{
GERALD BERMAN AND ROBERT J. SILVERMAN
}

1. Introduction. Consider a group $B=(G,+)$ (not necessarily commutative) and $T(G)$, the set of transformations on $G$. Define addition (+) and multiplication $(\cdot)$ on $T(G)$ by

(1.1) $g(A+B)=g A+g B, g(A B)=(g A) B, g \in G, A, B \in T(G)$.

Then $(T(G),+, \cdot)=\mathfrak{T}(\mathfrak{B})$ is a near-ring, the near-ring of transformations on (5). That is (i) $(T(G),+)$ is a group, (ii) $(T(G), \cdot)$ is a semigroup, and (iii) multiplication is left distributive with respect to addition:

$$
A(B+C)=A B+A C, \quad A, B, C \in T(G) .
$$

The transformation 0 , where $g 0=0$, for all $g \in G$, is the zero of $\mathfrak{T}(\$)$. Let $T_{0}(G)$ be the set of all transformations which commute with the zero transformation, i.e. $0 A=0, A \in T(G) . T_{0}(G)$ determines a sub-near-ring $\mathfrak{T}_{0}(\mathfrak{B})$ of $\mathfrak{T}(\mathfrak{S})$.

The main theorem is now stated.

Theorem 1. For any group $\mathfrak{( 5 ,} \mathfrak{T}(\mathfrak{S})$ and $\mathfrak{I}_{0}(\mathfrak{S})$ are simple.

That is, they have no proper nontrivial homomorphic images.

2. Preliminaries. A subset $Q$ of a near-ring $\mathfrak{B}$ determines an ideal of $\mathfrak{P}$ if and only if

(a) $(Q,+)$ is a normal subgroup of $(P,+)$,

(b) $P Q \subset Q$,

(c) $(a+q) b-a b$ is in $Q$ for all $a, b \in P, q \in Q$.

As in ring theory, the kernal $\mathfrak{Q}$ of a homomorphism $\theta$ from a nearring $\mathfrak{P}$ to a near-ring $\mathfrak{B}^{\prime}$ (i.e. the inverse image of the zero of $P^{\prime}$ ) is an ideal. Every ideal $\mathfrak{Q}$ is the kernal of the natural homomorphism $\nu: a \nu=a+Q$, from $\mathfrak{P}$ to the difference near-ring $\mathfrak{B}-\mathfrak{O}$, and every homomorphic image $\mathfrak{P} \theta$ with kernel $\mathfrak{Q}$ is isomorphic to $\mathfrak{P}-\mathfrak{Q}$. Thus a near-ring $\mathfrak{P}$ is simple if and only if its only ideals are itself and the zero ideal.

By way of warning, the following three concepts are introduced. A subset $Q$ of a near-ring $\mathfrak{B}$ determines $(\alpha)$ a left ideal if it satisfies $(\mathrm{a})$ and $(\mathrm{b}),(\beta)$ a right ideal if it satisfies (a) and $\left(\mathrm{b}^{\prime}\right) Q P \subset Q,(\gamma)$ a two-sided ideal if it satisfies (a), (b) and $\left(\mathrm{b}^{\prime}\right)$. While an ideal is a left ideal, examples show that ideals need not be two-sided ideals, and

Received by the editors April 17, 1958 and, in revised form, October 27, 1958. 
that two-sided ideals need not be ideals.

In the near-ring $\mathfrak{T}(\mathfrak{S})$, denote by $Z_{g}$ the transformation such that $g^{\prime} Z_{g}=g, g^{\prime} \in G$. Clearly $A Z_{g}=Z_{g}$, and $Z_{g} A=Z_{g A}$, for every $A$ in $T(G)$ and $g$ in $G$. In particular $Z_{0}=0$ in $(T(G),+)$.

Lemma 1. If $\mathfrak{Q}$ is an ideal of $\mathfrak{T}(\$)$, then $Q T_{0}(G) \subset Q$.

From (c), letting $a \in Q, b \in T_{0}(G),\left(Z_{0}+a\right) b-Z_{0} b=a b \in Q$. In particular, if $\mathfrak{Q}$ is an ideal of $\mathfrak{I}_{0}(\mathfrak{\$})$, then $\mathfrak{Q}$ is a two-sided ideal of $\mathfrak{I}_{0}(\mathfrak{\$})$.

LEMMA 2. The only sub-near-ring of $\mathfrak{T}(\mathfrak{B})$ which contains $\mathfrak{I}_{0}(\mathbb{S})$ properly is $\mathfrak{T}(\mathfrak{B})$ itself.

Let $\mathfrak{P}$ be a sub-near-ring of $\mathfrak{I}(\mathfrak{S})$ containing $\mathfrak{I}_{0}(\mathfrak{S})$. Consider $A \in P, A \in T_{0}(G)$. Then $A-Z_{0 A} \in T_{0}(G)$. Hence $Z_{0 A} \in P$. Since $0 A \neq 0$; and $Z_{0 A} B=Z_{0(A B)}$, and $T_{0}(G)$ is transitive on the nonzero elements of $G$, the set of $Z_{g}$ 's, $g \in G$ is in $P$. Thus, $C \in T(G), C$ $=\left(C-Z_{0 C}\right)+Z_{0 C}$ is in $P$, for $C-Z_{0 C}$ is in $T_{0}(G)$.

3. Proof of the theorem. A transformation $A \in T(G)$ has rank $R(A)=R$ if the set $\{g A \mid g \in G\}$ has cardinality $R$.

Lemma 3. A nonzero ideal $\mathfrak{\Im} \mathfrak{I}_{0}(\mathfrak{S})$ contains all the elements of rank 2.

By Lemma $1, \Im$ is a two-sided ideal, and since $\Im \neq\{0\}$, there exists a $V \in I$ and $g_{1}, g_{1}^{\prime} \in G, g_{1}^{\prime} \neq 0$ such that $g_{1} V=g_{1}^{\prime}$. Partition $G$ into disjoint sets $G_{1}$ and $G_{2}, 0 \in G_{2}$. Define $A \in T_{0}(G)$ such that $g A=g_{1}, g \in G_{1}, g A=0, g \in G_{2}$. Let $g^{\prime \prime}$ be any element of $G$, and let $B \in T_{0}(G)$ be such that $g_{1}^{\prime} B=g^{\prime \prime}$. Then $g A V B=g^{\prime \prime}, g \in G_{1}, g A V B=0$, $g \in G_{2}$, and $A V B \in I$.

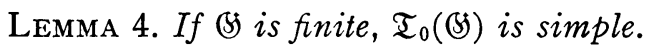

Suppose $I$ contains all elements of rank less than or equal to $k$. Partition $G$ into pairwise disjoint nonempty sets $G_{0}, G_{1}, \cdots, G_{k}$, $0 \in G_{0}$. Consider $k+1$ elements in $G, g_{0}=0, g_{1}, \cdots, g_{k}$. Define $A \in I$ such that $g A=g_{i}, g \in G_{i}, i=0,1, \cdots, k-1 ; g A=0, g \in G_{k}$. Define $B \in T_{0}(G)$ such that $g B=0, g \in G_{i}, i=0,1, \cdots, k-1, g B=g_{k}$, $g \in G_{k}$. Hence $C=A+B \in I$ and has rank $k+1$. Since the sets $G_{i}$, $i=0,1, \cdots, k$, and the elements $g_{i}, i=1,2, \cdots, k$ are arbitrary, $I=T_{0}(G)$ by induction.

Lemma 5. Let (5) have infinite cardinality and let $h \in G, h \neq 0$. Then there exists a maximal set $A \subset G$ such that $A \cap(A+h)=\varnothing$. Further, $A \cup(A+h) \cup(A-h)=G$. Hence the cardinality of $A, A+h$, and $A-h$ are each equal to the cardinality of $G$. 
Consider the collection of subsets of $G: S=\{S \mid(S+h) \cap S=\varnothing\}$. The collection $S$ is not empty since $\{0\} \in S$. Define a partial ordering $S_{1}>S_{2}$, if $S_{1} \supset S_{2}$, and $S_{1}, S_{2} \in \mathcal{S}$. Consider a linearly ordered subcollection $\left\{S_{t} \mid t \in T, S_{t} \in \mathcal{S}\right\}$. Then, it is asserted that $S^{\prime}=\bigcup_{t \in T} S_{t} \in \mathcal{S}$ and $S^{\prime}>S_{t}, t \in T$. Trivially, $S^{\prime} \supset S_{t}, t \in T$. Suppose $s^{\prime}=s^{\prime \prime}+h, s^{\prime}$, $s^{\prime \prime} \in S^{\prime}$. But, $s^{\prime}, s^{\prime \prime} \in S_{t}$ for some $t$, a contradiction. Hence by Zorn's lemma a maximal set $A$ exists.

Let $k \in G, k \in A, k \in A+h$. (If no such element $k$ exists, then $G=A \cup(A+h)$ and the sets $A$ and $A+h$ each have the cardinality of $G$.) The elements $k+h=a \in A$. For if not, consider $A^{\prime}=\{A, k\}$. Then $A^{\prime}+h$ is disjoint from $A^{\prime}$, contrary to the maximality of $A$. Therefore $k=a-h \in A-h$. Since $A, A+h, A-h$ have the same cardinality and their union is $G$, the lemma is proved.

LEMMA 6. If I contains a transformation of rank $d$, then I contains every transformation of rank less than or equal to $d$.

Let $\left\{G_{x} \subset G \mid x \in X\right\}$ be any partition of $G$ into pairwise disjoint sets, where $X$ is an index set of cardinality $d$, with $0 \in G_{x_{0}}$. Consider any collection $\left\{g_{x}^{\prime} \in G \mid x \in X, g_{x_{0}}^{\prime}=0\right\}$. Let $V \in I$ have rank $d$ and denote the elements in the image $G V$ of $V$ by $\left\{g_{x} \mid x \in X, g_{x_{0}}=0\right\}$. For each $g_{x} \in G V$, let $g_{x}^{\prime \prime}$ be an element such that $g_{x}^{\prime \prime} V=g_{x}$. Define $A \in T_{0}(G)$ such that $g A=g_{x}^{\prime \prime}, g \in G_{x}, x \in X$. Let $B$ be any element in $T_{0}(G)$ such that $g_{x} B=g_{x}^{\prime}$. Then $A V B$ is an arbitrary transformation of rank less than or equal to $d$ and is in $I$.

Lemma 7. If (\$) has infinite cardinality $\mathfrak{I}_{0}(\$)$ is simple.

Define the transformation $D_{h} \in T_{0}(G)$ by $g D_{h}=h, g \neq 0, g \in G$. Then by Lemma $3, D_{h} \in I$. Define $C \in T_{0}(G)$ by $g C=g, g \in A ; g C=0$, $g \notin A$, where $A$ is a maximal set (Lemma 5) such that $A \cap(A+h)=\varnothing$. Then $T=\left(1+D_{h}\right) C-C \in I$, where 1 is the identity map. Observe that $g T=(g+h) C-g C=-g, g \in A$. Hence $T$ has rank of the same cardinality as $G$. Thus, by the previous lemma, $I=T_{0}(G)$. It is only in Lemma 7 that the invariance property of an ideal is used in proving the simplicity of $\mathfrak{I}_{0}(\mathfrak{S})$.

LEMMA 8. T(\$) is simple.

If $G$ has order 2, then the theorem is easily checked directly. Assume therefore that $G$ has order greater than 2 . If $\Im$ is a nonzero ideal in $\mathfrak{I}(())$, and if there exists a $C \in I \cap T_{0}(G), C \neq 0$, then $T_{0}(G) \subset I$ by Lemmas $1,4,7$. In addition, since an ideal is a left ideal, $Z_{g} C=Z_{g C} \in I$, $g \in G$. Choose $g \in G$ so that $g C \neq 0$. Then, since the smallest near-ring properly containing $\mathfrak{T}_{0}(\mathfrak{S})$ is $\mathfrak{T}(\mathfrak{S})$, the lemma follows. Finally if the ideal contains no nonzero element of $T_{0}(G)$, consider $B \neq 0 \in I$ and 
$g \in G$ such that $g B=g_{1} \neq 0$. Then $Z_{g} B=Z_{g_{1}} \in I$. Let $C \in T_{0}(G)$ such that $g_{1} C=0$ and $\left(g_{2}+g_{1}\right) C \neq g_{2} C$ for some $g_{2} \in G, g_{2} \neq g_{1}, g_{2} \neq 0$. Then $D=\left(1+Z_{g_{1}}\right) C-C \in I$. Further $D \in T_{0}(G)$ and $g_{2} D \neq 0$, contrary to the assumption.

The theorem follows from Lemmas $4,7,8$.

4. Two-sided invariant sub-near-rings. A sub-near-ring $\mathfrak{Q}$ of a near-ring $\mathfrak{B}$ is two-sided invariant if conditions (b) and $\left(\mathrm{b}^{\prime}\right)$ of $\$ 2$ hold.

Lemma 9. The sets (i) $T_{z}(G)=\{A \in T(G) \mid R(A)=1\}$, (ii) $T_{f}(G)$ $=\left\{A \in T(G) \mid R(A)<\boldsymbol{\aleph}_{0}\right\}$, (iii) $T_{\aleph_{k}}(G)=\left\{A \in T(G) \mid R(A) \leqq \boldsymbol{\aleph}_{k}\right\}$, where $\boldsymbol{\aleph}_{k}$ is an infinite cardinal number, determine two-sided invariant subnear-rings of $\mathfrak{T}(\mathfrak{S})$. Further $T \boldsymbol{\aleph}_{k_{1}}(G) \supsetneqq T \aleph_{k_{2}}(G)$ provided $d \geqq \boldsymbol{\aleph}_{k_{1}}>\boldsymbol{\aleph}_{k_{2}}$, where $d$ is the cardinality of $T(G)$.

The proof of this lemma is straight forward and depends on very simple properties of cardinal numbers. The theorem which follows shows that these near-rings determine the two sided invariant subnear-rings of $\mathfrak{T}(\mathfrak{S})$ and $\mathfrak{T}_{0}(\mathfrak{S})$.

THEOREM 2. (i) The two-sided invariant sub-near-rings of $\mathfrak{T}(\mathfrak{S})$ are $\mathfrak{I}_{z}(\mathfrak{S}), \mathfrak{I}_{f}(\mathfrak{S})$, and $\mathfrak{I N}_{k}(\mathfrak{\$})$ for any infinite cardinal number $\boldsymbol{\aleph}_{k}$ less than or equal to the cardinality of (5). (ii) The two-sided invariant subnear-rings of $\mathfrak{T}_{0}(\mathfrak{S})$ are $\{0\}$ and the intersection of the two-sided invariant sub-near-rings of $\mathfrak{T}(\mathbb{S})$ with $\mathfrak{T}_{0}(\mathbb{S})$.

Let $\mathfrak{P}$ be a two-sided invariant sub-near-ring of $\mathfrak{T}(\mathfrak{S})$ which has an element $C$ of maximal infinite rank $\boldsymbol{\aleph}_{k}$. Then, for suitable choices of $A, B \in T(G), A C B$ represents any transformation whose rank is less than or equal to $\boldsymbol{\aleph}_{k}$. (See proof of Lemma 6.) Thus $\mathfrak{B}=\mathfrak{T} \boldsymbol{N}_{k}(\mathfrak{S i})$. If $P$ contains no element of infinite rank, suppose $P$ contains an element $C$ of rank greater than 1 . Then as above it follows that every element of rank less than or equal to the rank of $C$ is in $P$. The argument used in the proof of Lemma 4 is now valid to show that $\mathfrak{P}$ $=\mathfrak{I}_{f}(\mathfrak{S})$. If the rank of every element of $P$ is 1 , then $P \subset T_{z}(G)$ and it is immediate that $\mathfrak{B}=\mathfrak{T}_{z}(\mathfrak{S})$. The proof of (ii) is similar.

\section{REFERENCES}

1. Gerald Berman and Robert J. Silverman, Near-rings, Amer. Math. Monthly vol. 66 (1959) pp. 23-34.

2. D. W. Blackett, Simple and semi-simple near-rings, Proc. Amer. Math. Soc. vol. 4 (1953) pp. 772-785.

3. L. E. Dickson, On finite algebras, Nachr. Akad. Wiss. Göttingen (1905) pp. 358-393.

4. H. Zassenhaus, Über enliche Fastkörper, Abh. Math. Sem. Univ. Hamburg. vol. 11 (1936) pp. 187-220. 